\title{
Spontaneous Chiral Symmetry Breaking as Condensation of Dynamical Chirality
}

\author{
Andrei Alexandru ${ }^{1}$ and Ivan Horváth ${ }^{2}$ \\ ${ }^{1}$ The George Washington University, Washington, DC, USA \\ ${ }^{2}$ University of Kentucky, Lexington, KY, USA
}

Nov 142012

\begin{abstract}
The occurrence of spontaneous chiral symmetry breaking (SChSB) is equivalent to sufficient abundance of Dirac near-zeromodes. However, dynamical mechanism leading to breakdown of chiral symmetry should be naturally reflected in chiral properties of the modes. Here we offer such connection, presenting evidence that SChSB in QCD proceeds via the appearance of modes exhibiting dynamical tendency for local chiral polarization. These modes form a band of finite width $\Lambda_{c h}$ (chiral polarization scale) around the surface of otherwise anti-polarized Dirac sea, and condense. $\Lambda_{c h}$ characterizes the dynamics of the breaking phenomenon and can be converted to a quark mass scale, thus offering conceptual means to determine which quarks of nature are governed by broken chiral dynamics. It is proposed that, within the context of SU(3) gauge theories with fundamental Dirac quarks, mode condensation is equivalent to chiral polarization. This makes $\Lambda_{c h}$ an "order parameter" of SChSB, albeit without local dynamical field representation away from chiral limit. Several uses of these features, both at zero and finite temperature, are discussed. Our initial estimates are $\Lambda_{c h} \approx 150$ $\mathrm{MeV}\left(N_{f}=0\right), \Lambda_{c h} \approx 80 \mathrm{MeV}\left(N_{f}=2+1\right.$, physical point $)$, and that the strange quark is too heavy to be crucially influenced by broken chiral symmetry.
\end{abstract}

1. Introduction and Conclusions. Chiral symmetry and its conjectured dynamical breaking pattern in multi-flavor massless QCD is a crucial ingredient in the current understanding of low energy hadronic physics. While SChSB scenario is widely accepted, and there is no first-principles evidence suggesting otherwise, it is not known how strong interactions induce its vacuum to become a non-symmetric state. To help the identification of the corresponding mechanism, it is desirable to search for dynamical circumstances accompanying the phenomenon, i.e. to find dynamical features that the eventual explanation needs to incorporate. Low-lying Dirac modes are a suitable place to look for such signatures since they encode the nature of quark propagation in the chiral regime as well as the condensate itself. Indeed, the most direct expression of this connection is the Banks-Casher relation, revealing that the symmetry breakdown is equivalent to sufficient accumulation of Dirac near-zeromodes [1. However, being entirely generic, the Banks-Casher relation doesn't shed light on dynamical specifics of SChSB.

The premise of this work is that dynamical features of the theory relevant to chiral symmetry breaking should be imprinted in chiral properties of Dirac eigenmodes. While there cannot be any average preference for left or right, QCD dynamics induces specific 

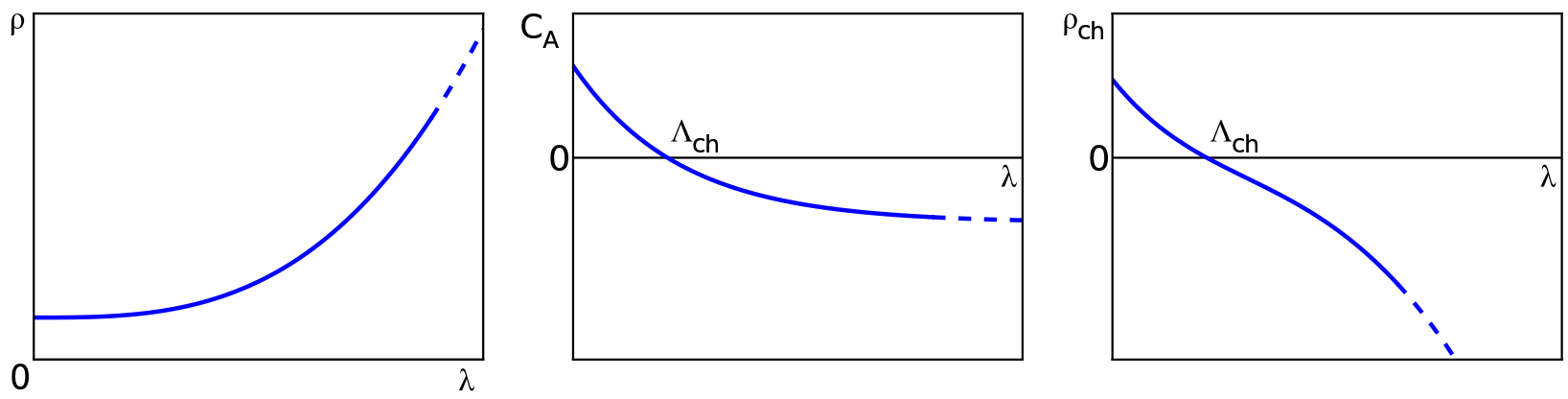

Figure 1: Schematic behavior of $\rho(\lambda), C_{A}(\lambda), \rho_{c h}(\lambda)$ in theory with Dirac mode condensation.

chiral properties in the eigenmodes locally. The most basic of these describe whether values $\psi(x)=\psi_{L}(x)+\psi_{R}(x)$ tend to involve asymmetric participation of left-right subspaces (chiral polarization) as opposed to equal participation (chiral anti-polarization). Building on the earlier local chirality approach of Ref. [2], proper dynamical quantifiers of this type have recently been constructed [3].

Dynamical nature of these new polarization measures stems from the fact that they are defined relative to the case of statistically independent left-right components, and thus represent (uniquely constructed) correlations. In this work we will only be concerned with the overall dynamical tendency described by the correlation coefficient of chiral polarization $C_{A}$. Let $\mathcal{P}\left(\psi_{L}, \psi_{R}\right)$ be the probability distribution defined by the collection of values comprising given mode(s), and $\mathcal{P}^{u}\left(\psi_{L}, \psi_{R}\right)$ the associated distribution of statistically independent components. If $\Gamma_{A}$ is the probability that a sample chosen from $\mathcal{P}$ is more polarized than the sample chosen from $\mathcal{P}^{u}$, then $C_{A} \equiv 2 \Gamma_{A}-1 \in[-1,1]$. Thus, the enhancement of polarization relative to statistical independence $\left(\Gamma_{A}>1 / 2\right)$ is associated with correlation $\left(C_{A}>0\right)$ while its suppression $\left(\Gamma_{A}<1 / 2\right)$ with anti-correlation $\left(C_{A}<0\right)$.

The claims in this work are mostly based on the proposed behavior of the QCD average for $C_{A}$ in modes at eigenvalue $\lambda$. In particular, for quark setups relevant to real world, such as $N_{f}=2+1$ at zero temperature, we conclude the behavior shown in Fig. 1 (middle) at generic quark masses: there is a low-lying band of chirally polarized modes separated from the rest of anti-polarized bulk. The suggestion is that this applies in the infinite volume and also when light quark masses are asymptotically small, with chiral polarization scale $\Lambda_{c h}$, introduced in Ref. [3], remaining strictly positive1 1 Since broken chiral dynamics of quarks is dominated by near-zeromodes, we are proposing that this dynamics (1) is associated with chirally polarized (correlated) modes, and that (2) the mechanism generating the polarized band involves a dynamical scale $\Lambda_{c h}$. These definite properties need to be respected by viable models of SChSB, exemplifying a "bottom-up" approach to QCD vacuum structure [4].

Thus, our Conjecture 1 implies that the chirality-related dynamical feature of SChSB in QCD is that its condensing modes are locally polarized. How important is this in the context of the mechanism generating broken chiral dynamics? One way to approach this is to consider a wider range of theories, governed by the same gauge interaction, and ask whether the presence of broken chiral dynamics is equivalent to chiral polarization, i.e.

\footnotetext{
${ }^{1}$ Chiral polarization scale was denoted $\Lambda_{T}$ in Ref. 3]. Here we switch to $\Lambda_{c h}$ so that the label is not confused with temperature.
} 
whether SChSB occurs only in conjunction with chiral polarization and vice versa. Indeed, if such correspondence holds, then chiral polarization is intimately tied to the nature of the interaction. For purposes of this discussion, we will use the context of $\mathrm{SU}(3)$ gauge theories with arbitrary number $\mathrm{N}_{f}$ of Dirac fermions in the fundamental representation, and at arbitrary temperatures. Our Conjecture 2 implies that the above equivalence holds.

Accepting the relevance of chiral polarization elevates $\Lambda_{c h}$ into a scale associated with SChSB. This input can be used to define the scale of SChSB in a more conventional language of quark mass. Resulting chiral polarization mass scale $m_{c h}$ represents maximal valence mass at which spectral components of the associated scalar bilinear favor chiral polarization over chiral anti-polarization on average. From chiral standpoint, quark dynamics at lower mass is similar to massless one, while that at higher mass turns qualitatively different. Moreover, $\Lambda_{c h}$ and $m_{c h}$ are meaningful even when all quarks are massive, and some of them can still be driven by broken-like dynamics if their mass is lower than theory's $m_{c h}$. Thus, one utility of the above dynamical insight is a possibility of objective labeling nature's quarks as "light" or "heavy". These dynamical features also put a novel angle on the characterization of strong dynamics at finite temperature, as we discuss in some detail.

The proposed connection between SChSB and dynamical chirality is associated with the following narrative. Viewing Dirac eigenmodes as scale-dependent probes of gauge field, their non-interacting baseline is a perfectly anti-polarized state. Indeed, left and right components of free eigenmodes have identical magnitudes, independently of $\lambda$ in this scaleinvariant situation, and there is no mode condensation. Turning on the interaction among gluons (pure glue QCD) works against anti-polarization. This effect is scale dependent, due to running coupling, with modes in the infrared being affected more than those in the ultraviolet. At low energy the interaction becomes sufficiently strong for the chiral behavior of modes to undergo a qualitative change: scale $\Lambda_{c h}$ is dynamically generated via appearance of chirally polarized, condensing Dirac modes, while logarithmically violated scale invariance remains in the ultraviolet. The above two cases (free and pure glue) represent chiral extremes, with other gauge-quark setups interpolating between them. Indeed, both light dynamical quarks and the temperature tend to reduce the effective gauge coupling at low energy, and thus possibly destroy chiral polarization relative to pure glue. Our conclusion in most general form is that the Dirac mode condensation and chiral polarization are present/absent simultaneously: chiral polarization scale $\Lambda_{c h}$ is an indicator of mode condensation and, when some of the quarks are massless, an "order parameter" of SChSB.

2. Definitions and Claims. Here we specify our main conclusions more precisely. The setup and notation of continuum Euclidean QCD is used for simplicity, but it should be understood that the concepts below have well-defined meaning acquired via lattice regularization respecting chirality, e.g. lattice QCD with overlap fermions [5]. Thus, the spectrum of continuum massless Dirac operator in a given gauge background is located on imaginary axis, and the zero-temperature theory with $\mathrm{N}_{f}$ flavors of quarks can be labeled by masses $M \equiv\left(m_{1}, m_{2}, \ldots, m_{N_{f}}\right)$. Ordering the eigenmodes $\psi_{k}(x)$ by magnitude of their eigenvalue $i \lambda_{k}$, the average correlation of chiral polarization for modes at $i \lambda$ reads

$$
C_{A}(\lambda, M, V) \equiv \frac{\sum_{k}\left\langle\delta\left(\lambda-\lambda_{k}\right) C_{A, k}\right\rangle_{M, V}}{\sum_{k}\left\langle\delta\left(\lambda-\lambda_{k}\right)\right\rangle_{M, V}}=\frac{\rho_{c h}(\lambda, M, V)}{\rho(\lambda, M, V)}
$$


Here $\langle\ldots\rangle_{M, V}$ denotes QCD expectation value in 4 -volume $V, C_{A, k}$ is the correlation associated with mode $\psi_{k}$, and we have introduced the spectral chiral polarization density

$$
\rho_{c h}(\lambda, M, V) \equiv \frac{1}{V} \sum_{k}\left\langle\delta\left(\lambda-\lambda_{k}\right) C_{A, k}\right\rangle_{M, V}
$$

in addition to spectral mode density $\rho(\lambda, M, V)$. Correlation $C_{A, k}$ can be evaluated relative to mode's own distribution $\mathcal{P}_{k}^{u}$ of independent left-right components, or relative to $\mathcal{P}_{\lambda}^{u}$ involving all modes at eigenvalue $i \lambda$. These definitions are expected to be equivalent in the infinite volume limit. To include setups at finite temperature $T$ one simply replaces labels $V \rightarrow T, V_{3}$ and factors $1 / V \rightarrow T / V_{3}$, where $V_{3}$ is a 3 -volume.

There are two reasons for introducing $\rho_{c h}$. First, spectral contribution to fermionic bilinears is proportional to spectral mode density, and thus $\rho C_{A}=\rho_{c h}$ gives the proper spectral weight to local chirality in that context. Secondly, while $C_{A}(\lambda)$ is not defined in potential regions of spectral exclusion, i.e. parts of real axis with no eigenvalues in any background, $\rho_{c h}(\lambda)$ assumes definite value for each $\lambda$. Note that, when $\rho(\lambda)$ is non-zero, $C_{A}(\lambda)$ and $\rho_{c h}(\lambda)$ have identical signs and zeroes, as seen e.g. in Fig. 1 (right).

Theory is said to exhibit "mode condensation" if $\lim _{\lambda \rightarrow 0} \lim _{V \rightarrow \infty} \rho(\lambda, M, V) \equiv \rho(0, M)>0$, with obvious generalization to finite temperature. Similarly, "chirality condensation" occurs when $\rho_{c h}(0, M)>0$, and "anti-chirality condensation" when $\rho_{c h}(0, M)<0$. By "QCD" we mean SU(3) gauge theories in three spatial dimensions with Dirac quarks in the fundamental representation. The statements below are formulated in infinite volume where the concepts of spontaneous symmetry breaking and condensation assume their meaning.

Conjecture 1: Consider zero-temperature $Q C D$ with $N_{f}=2+1$ flavors of quarks, i.e. $M=$ $\left(m_{l}, m_{l}, m_{h}\right), m_{l} \in[0, \infty], m_{l}<m_{h}$. For every $M$ there exists $\Lambda_{c h} \equiv \Lambda_{c h}(M)>0$ such that functions $\rho_{c h}(\lambda)$ and $C_{A}(\lambda)$ are positive on $\left[0, \Lambda_{c h}\right)$ and negative on $\left(\Lambda_{c h}, \infty\right)$.

Note that the above statement subsumes the standard expectation that Dirac modes in $\mathrm{N}_{f}=2+1$ QCD always condense. Indeed, since $\left|\rho_{c h}(\lambda)\right| \leq \rho(\lambda)$, it follows that $\rho(0)>0$ for all $M$. The novelty is in the claim that this condensation is always associated with chirally polarized modes and non-zero $\Lambda_{c h}$, including in the massless $\left(m_{l}=0\right)$ limit. The latter makes this behavior a dynamical feature of SChSB in QCD. Similar conclusion is expected to hold in other physically relevant cases such as $\mathrm{N}_{f}=2$ or $\mathrm{N}_{f}=2+1+1+1+1$.

Conjecture 2: Consider $Q C D$ theories with arbitrary $N_{f} \geq 0$ quark flavors of arbitrary masses $M$, at arbitrary temperature $T$. Within this set, $\rho(0, M, T)>0$ if and only if there exists $\Lambda_{c h} \equiv \Lambda_{c h}(M, T)>0$ such that $\rho_{c h}(\lambda)$ and $C_{A}(\lambda)$ are positive on $\left[0, \Lambda_{c h}\right)$ and negative on $\left(\Lambda_{c h}, \infty\right)$. Moreover, $\rho(0, M, T)=0$ if and only if $\rho_{c h}(\lambda) \leq 0$ on $[0, \infty)$.

We thus propose that, in the context of $\mathrm{SU}(3)$ gauge theories with fundamental quarks, Dirac mode condensation is synonymous with chiral polarization in the above sense, including in chiral corners of the theory space. The statement implies that there are two possible behaviors of $\rho_{c h}(\lambda)$ : either there is anti-polarization over the whole spectrum or there exists a finite layer of polarization around the surface of otherwise anti-polarized Dirac sea. The associated two classes of theories correspond to those defined by absence/presence of Dirac mode condensation. We wish to highlight the following corollary. 

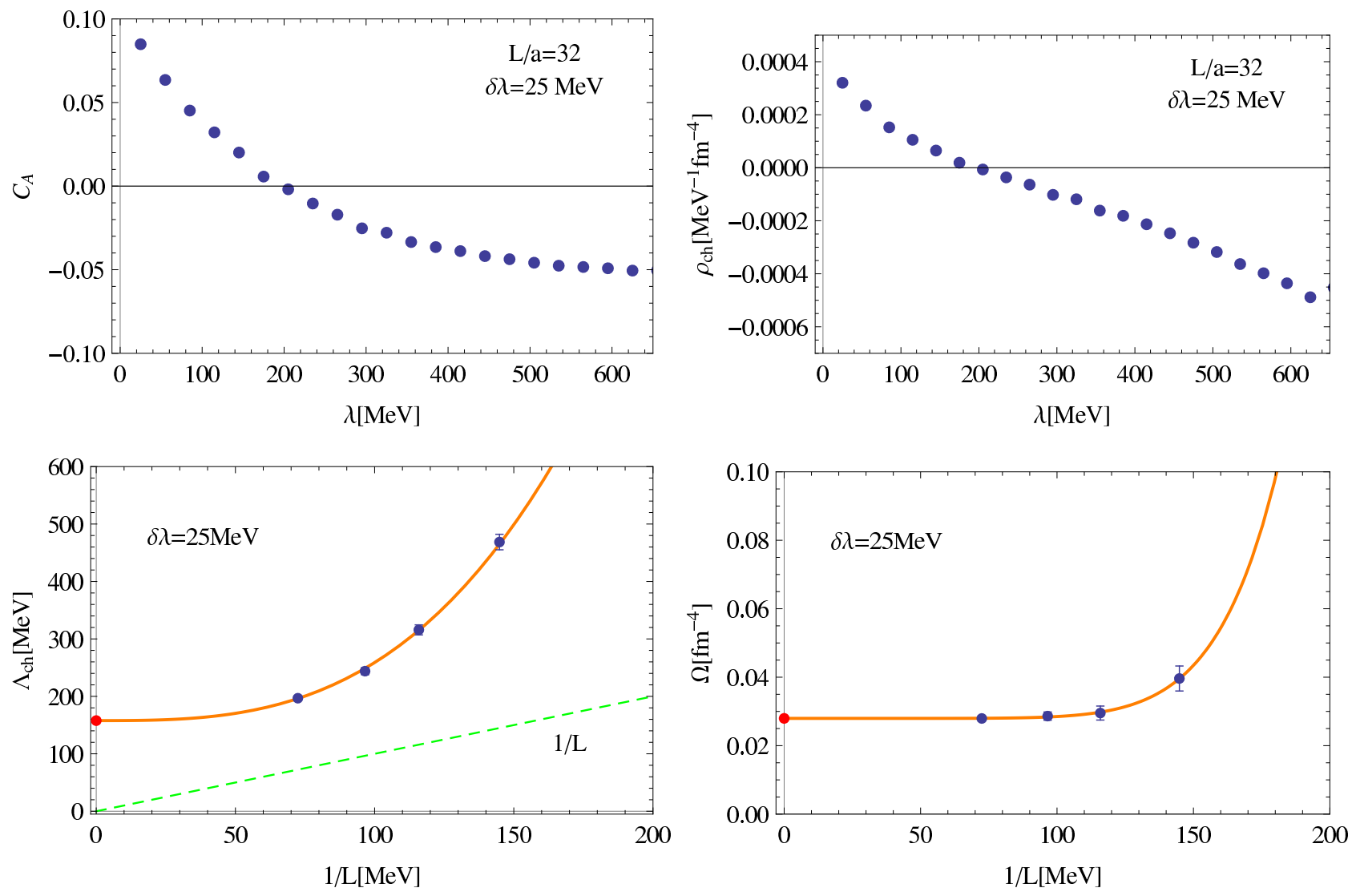

Figure 2: Top: behavior of $C_{A}(\lambda)$ and $\rho_{c h}(\lambda)$ in quenched QCD, as discussed in the text. Bottom: infinite volume extrapolation of $\Lambda_{c h}$ (left) and $\Omega$ of Sec. 6 (right).

Corollary 1: Condensation of Dirac modes in QCD theories is a condensation of dynamical chirality, and so is SChSB. Anti-chirality doesn't condense.

We emphasize that the above statements should be understood as being valid in latticeregularized theory at sufficiently large cutoffs, with appropriate limiting procedures implied. The precise value of chiral polarization scale $\Lambda_{c h}$ for given continuum theory likely depends on the details of this regularization, and finite normalization procedure might be needed to make it unique. However, the properties discussed here only depend on $\Lambda_{c h}$ being positive, and are thus expected to be universal.

3. Chiral Polarization Scale. In the following few sections we present elements of evidence in support of the above statements. In all of our calculations we analyze the eigenmodes of overlap Dirac operator (parameters $r=1, \rho=26 / 19$ ) to ensure proper chirality properties at the regularized level. The band of chirally polarized low-energy modes in quenched QCD was found in Ref. [3]. This work also showed that the point in the Dirac spectrum separating polarization from anti-polarization, namely $\Lambda_{c h}$, is non-zero in the continuum limit at fixed physical volume. However, to be considered a true dynamical scale in the theory, $\Lambda_{c h}$ needs to be demonstrably distinct from infrared cutoff.

To supply this crucial element, we calculated $C_{A}(\lambda)$ and $\rho_{c h}(\lambda)$ in quenched QCD at fixed lattice cutoff and several volumes. In particular, we work with Wilson lattice gauge theory 

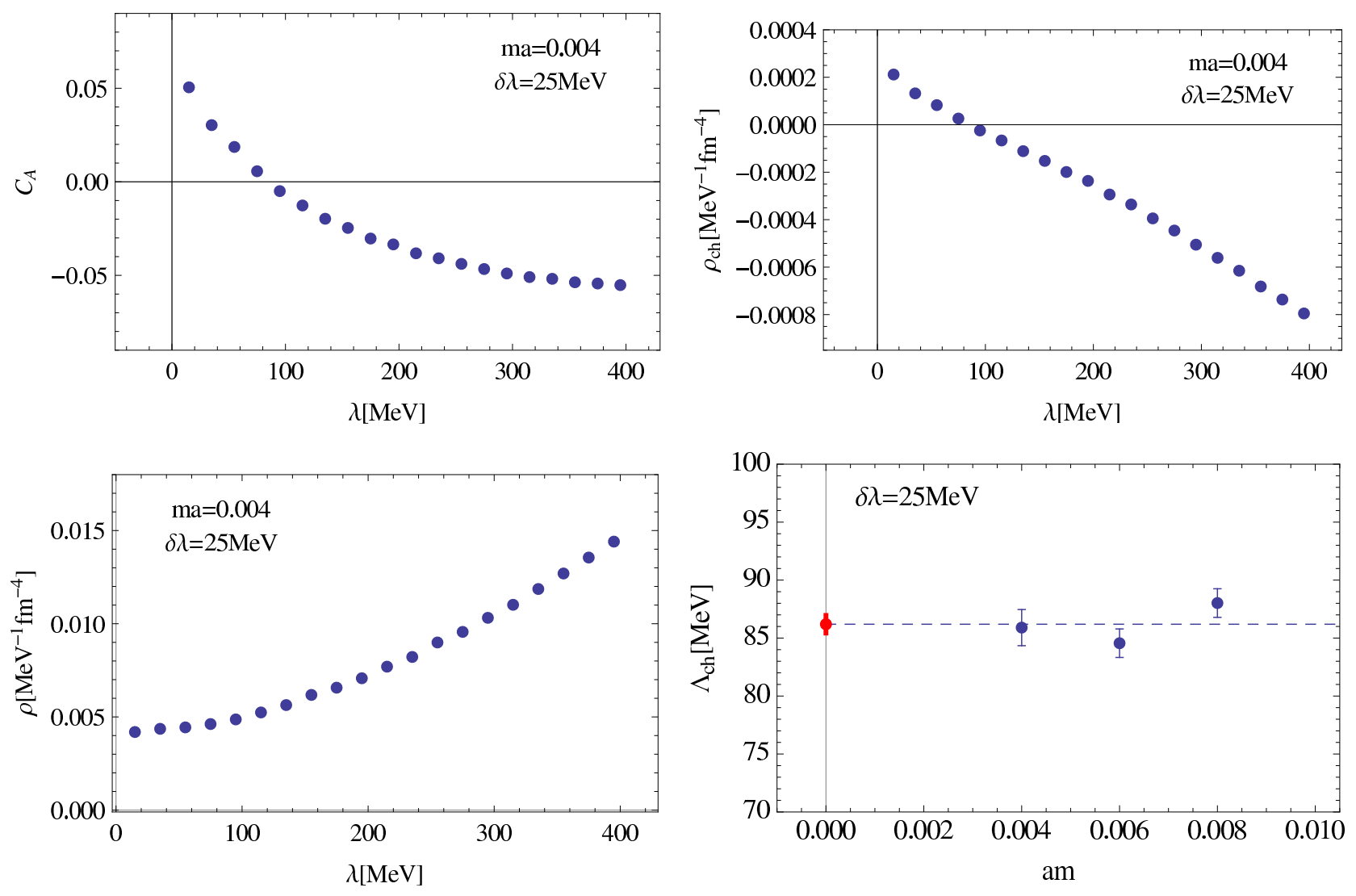

Figure 3: The behavior of $C_{A}(\lambda), \rho_{c h}(\lambda)$ and $\rho(\lambda)$ for the $m_{l} a=0.004$ dynamical ensemble (see text). The light mass dependence of $\Lambda_{c h}$ is shown in the lower right plot together with chiral extrapolation.

at $\beta=6.054$ which translates into lattice spacing $a=0.085 \mathrm{fm}$ when invoking reference scale $r_{0}=0.5 \mathrm{fm}$. Symmetric lattices of $N^{4}$ sites were used with $N=16,20,24,32$. Low-lying Dirac eigenmodes were computed on 100 equilibrated configurations for each system with $C_{A}(\lambda)$ and $\rho_{c h}(\lambda)$ evaluated using the modes in the strip $(\lambda-\delta \lambda / 2, \lambda+\delta \lambda / 2)$. We note that, in this regularized case, $\lambda$ refers to magnitude of the overlap eigenvalue. In Fig 2 (top) we show the result for the largest lattice, displaying the advertised behavior, including a clearly defined $\Lambda_{c h}$. The value of coarse graining parameter $\delta \lambda=25 \mathrm{MeV}$ is sufficiently small so that its variation induces only negligible changes in $\Lambda_{c h}$.

The volume dependence of $\Lambda_{c h}$ is shown on the lower left plot of Fig,2. The curvature of the data strongly suggests a positive infinite volume limit. Note that the infrared cutoff $1 / L$ is also shown. Chi-Squares for fits of the form $\Lambda_{c h}(1 / L)=\Lambda_{c h}(0)+b(1 / L)^{n}$, with integer $n$, revealed a deep narrow minimum at $n=3$. This fitting function was then used for the infinite volume extrapolation shown. Thus, with Wilson lattice regularization at $a=0.085$ fm, our calculation gives $\Lambda_{c h} \approx 160 \mathrm{MeV}$ in the infinite volume limit of quenched QCD. Using the continuum extrapolation of Ref. [3] as a rough guide for the size of cutoff effects, we estimate the continuum value to be $\Lambda_{c h} \approx 150 \mathrm{MeV}$ in this regularization. 

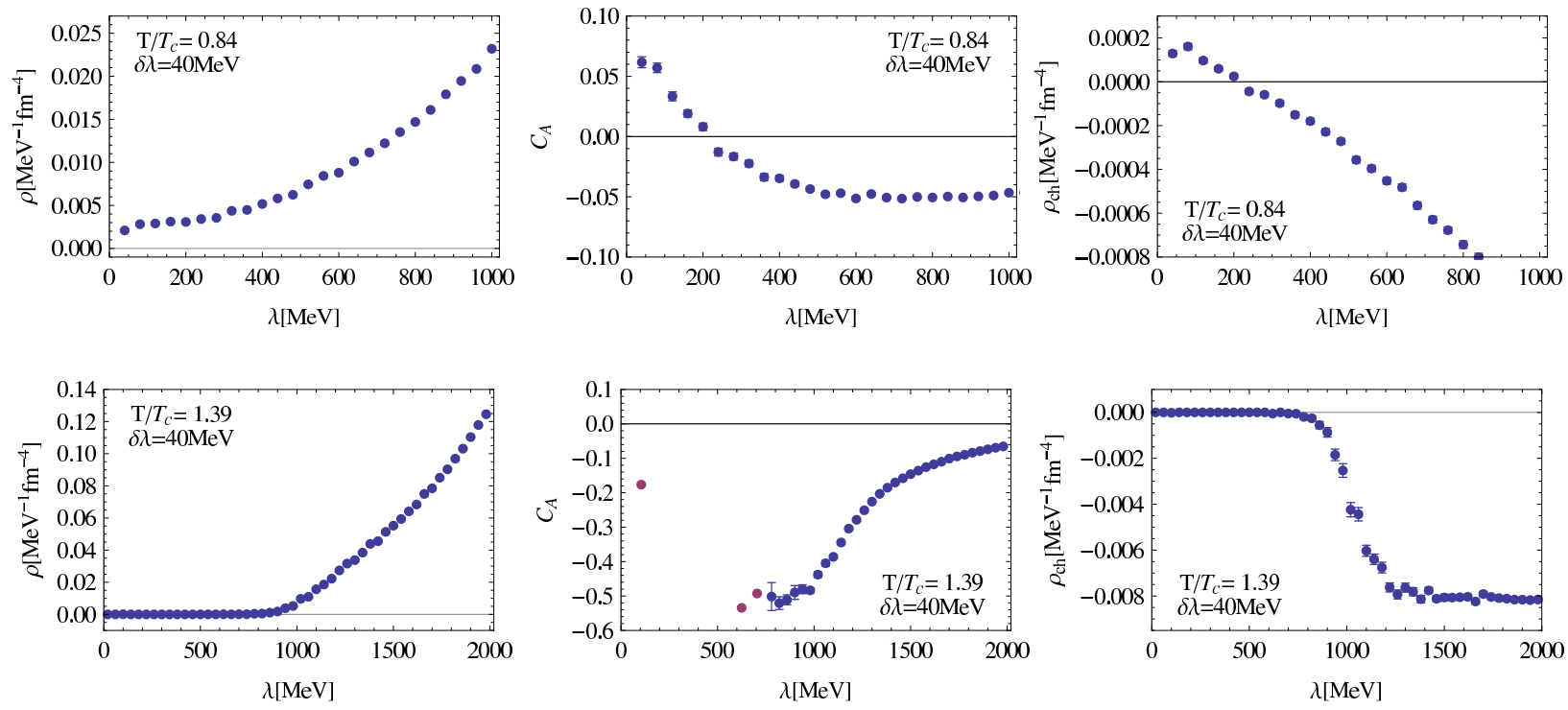

Figure 4: Top: functions $\rho(\lambda), C_{A}(\lambda)$ and $\rho_{c h}(\lambda)$ in quenched QCD at $T / T_{c}=0.84$, with 200 configurations. Bottom: same at $T / T_{c}=1.39$ with 100 configurations.

4. Dynamical Light Quarks. We now turn to the question whether light quarks in $N_{f}=$ $2+1$ QCD could destroy chiral polarization of low-lying Dirac modes, thus disqualifying it as a dynamical feature of SChSB. Advantage of lattice regularizations respecting chiral symmetry is that such checks are meaningful at fixed cutoff. In this work we use the $32^{3} \times 64$ domain wall (DW) ensembles generated by RBC/UKQCD collaborations as described in Ref. [6]. These three ensembles have fixed heavy bare mass $m_{h} a=0.03$ and lattice spacing $a=0.085 \mathrm{fm}$. The three light bare masses $m_{l} a=0.008,0.006,0.004$ correspond to pion masses $m_{\pi}=397,350$ and $295 \mathrm{MeV}$ respectively. Since the scale has been set using the physical value of the $\Omega$ baryon, the heavy quark can be considered to have approximately the mass of the strange quark.

We probe the vacua of the above DW lattice theories using the closely related overlap fermions. In particular, low-lying overlap Dirac eigenmodes were computed on 50 configurations from each ensemble and the characteristics of interest were evaluated. The functions $C_{A}(\lambda), \rho_{c h}(\lambda)$ and $\rho(\lambda)$ for ensemble with lightest quarks are shown in Fig. 3. Note that the behavior consistent with Conjecture 1 is obtained, with the value of $\Lambda_{c h}$ smaller relative to the quenched case, as expected. The important feature of our results is that the range of $m_{l}$ considered involves masses sufficiently small so that there is no appreciable change of $\Lambda_{c h}$ detected. This is shown on the lower right plot of Fig. 3 together with extrapolation to chiral limit via constant fit. We estimate $\Lambda_{c h} \approx 86 \mathrm{MeV}$ in the massless limit of this regularization, with the naive ( $N_{f}=0$ based) expectation of the continuum value to be $5-10 \%$ lower.

5. Finite Temperature. To check whether chiral polarization indeed goes hand in hand with mode condensation, as proposed in Conjecture 2, it is necessary to investigate systems in which the latter is expected to be absent. One way to do this is to turn on the temperature. For this purpose, we studied overlap Dirac eigenmodes on $20^{3} \times N_{t}$ lattices of quenched QCD. The setup is otherwise identical to that described in Sec. 3. In particular, the same (Wilson) action is used at identical gauge coupling, corresponding to $a=0.085 \mathrm{fm}$. 
Dirac mode condensation is expected to disappear in the vicinity of Polyakov line phase transition temperature $T_{c}$. Continuum-extrapolated value of $T_{c}$ is well known [7], and in Fig. 4 we show the relevant eigenmode characteristics at $T / T_{c}=0.84\left(N_{t}=10\right)$, and at $T / T_{c}=1.39\left(N_{t}=6\right)$. Following the standard practice in quenched theory [8, 9], we compute fermionic observables over configurations from the "real $Z_{3}$ " Polyakov line sector in order to facilitate smooth connection to simulations with dynamical quarks.

As expected, the behavior of $\rho(\lambda)$ at $T / T_{c}=0.84$ is consistent with mode condensation while that at $T / T_{c}=1.39$ with its absence. The associated $C_{A}(\lambda)$ reflects this with positive core of chiral polarization in the former case and the strictly negative values in the latter. Note that the red points in the plot for $T / T_{c}=1.39$ signify bins with too few eigenmodes for an error bar to be estimated. It is feasible that, in the limit of infinite statistics, $C_{A}(\lambda)$ is defined everywhere, and there is no true spectral exclusion region. Chiral density $\rho_{c h}(\lambda)$ is of course not affected by sparse occurrence of low-lying eigenmodes and exhibits a behavior described by Conjecture 2. Detailed account of these calculations, and of the correspondence, will be presented elsewhere [10].

6. The Uses: Order Parameters and Finite Volume. It is interesting to elaborate on some of the consequences stemming from the proposed connection of Dirac mode condensation to dynamical chiral polarization. To summarize the ingredients involved, consider the scalar fermionic density corresponding to valence quark mass $m_{v}$, namely

$$
S\left(m_{v}, M\right) \equiv\left\langle\operatorname{tr}\left(D+m_{v}\right)_{x, x}^{-1}\right\rangle_{M}=\int_{0}^{\infty} d \lambda \frac{2 m_{v}}{m_{v}^{2}+\lambda^{2}} \rho(\lambda, M)
$$

Thus, the scalar density associated with dynamical flavor $f$ is $\left\langle\bar{\psi} \psi_{f}\right\rangle_{M}=-S\left(m_{f}, M\right)$. We say that the theory exhibits "valence" SChSB, or broken valence chiral dynamics, if $\lim _{m_{v} \rightarrow 0} S\left(m_{v}, M\right)>0$. Let's further define the associated chiral scalar density via the spectral relation

$$
S_{c h}\left(m_{v}, M\right) \equiv \int_{0}^{\infty} d \lambda \frac{2 m_{v}}{m_{v}^{2}+\lambda^{2}} \rho_{c h}(\lambda, M)
$$

For both $S$ and $S_{c h}$ we have Banks-Casher type of relations, namely

$$
\lim _{m_{v} \rightarrow 0} S\left(m_{v}, M\right)=\pi \lim _{\lambda \rightarrow 0} \rho(\lambda, M) \quad \lim _{m_{v} \rightarrow 0} S_{c h}\left(m_{v}, M\right)=\pi \lim _{\lambda \rightarrow 0} \rho_{c h}(\lambda, M)
$$

with the former one expressing that Dirac mode condensation (at arbitrary $M$ ) is equivalent to valence SchSB. The equations above assume infinite volume.

Conclusions of this paper imply that $S(0, M), \rho(0, M), S_{c h}(0, M), \rho_{c h}(0, M)$ and $\Lambda_{c h}(M)$ can only be strictly positive simultaneously, thus extending the standard relation involving just $S$ and $\rho$. The appearance of chiral polarization scale in the above mix is conceptually interesting since it transforms Dirac mode condensation (and SChSB) from strictly infrared $(\lambda=0)$ effect into a feature that can be probed at scale $\Lambda_{c h}$. This suggests that the behavior in systems of size $1 / L \approx \Lambda_{c h}$ should be sufficient to indicate whether given theory is condensing. Note that, unlike the " $\lambda=0$ " quantifiers, the positivity of $\Lambda_{c h}$ is compatible with finite volume. Indeed, to arbitrary function $\rho_{c h}(\lambda)$ on $[0, \infty)$ for which there exist $\lambda_{1}<\lambda_{2}$ such that $\rho_{c h}(\lambda) \leq 0$ on $\left(\lambda_{1}, \lambda_{2}\right)$, we assign $\Lambda_{c h} \in[0, \infty)$ as the least $\lambda_{1}$ with that property. Chiral densities with $\Lambda_{c h}$ assigned in this manner are positive on $\left[0, \Lambda_{c h}\right)$ except 
for possible isolated zeros. Thus, even in finite volume, where $\rho_{\text {ch }}(0)=0$, scale $\Lambda_{\text {ch }}$ can be strictly positive, e.g. when $\rho_{c h}(\lambda)>0$ on $\left(0, \Lambda_{c h}\right)$. This was clearly seen in calculations presented in this work.

The above considerations make $\Lambda_{c h}(M)$ an attractive "order parameter" for valence $\mathrm{SChSB}$ in SU(3) gauge theories, and for a physical SChSB in chiral corners of the theory space. Potentially useful alternative serving the same purpose is given by

$$
\Omega(M) \equiv \lim _{\Lambda \rightarrow \Lambda_{c h}(M)^{+}} \int_{0}^{\Lambda} d \lambda \rho_{c h}(\lambda, M)
$$

i.e. total chiral polarization per unit volume. Note that $\Omega$ is a more general quantifier that would supersede $\Lambda_{c h}$ in unlikely situations with chiral polarization entering only via a positive $\delta(\lambda)$ term in $\rho_{c h}(\lambda)$. Indeed, this corresponds to $\Lambda_{c h}=0$ but $\Omega>0$. From practical standpoint it is important that $\Omega$ exhibits smaller finite volume effects than $\Lambda_{c h}$, as shown in Fig. 2 (bottom right).

7. The Uses: Broken Chiral Dynamics. Assuming Conjectures 1,2 withstand further scrutiny, the dynamical information they carry offers additional possibilities. Here we point out that, since broken chiral dynamics is associated with chiral polarization while the symmetric behavior with anti-polarization, one may characterize the situation for massive quark by the balance of the two tendencies in the associated scalar density. This is what $S_{c h}$ of Eq. (44) has been designed for. Indeed, the integrands of Eqs. (3]4), namely contributions of modes at value $\lambda$, are identical up to a factor $C_{A}$, since $\rho_{c h}=\rho C_{A}$. The meaning of $S_{c h}\left(m_{v}, M\right)$ is thus clarified by the fact that $S_{c h}\left(m_{v}, M\right) / S\left(m_{v}, M\right)$ expresses the average chiral polarization content of scalar density at valence mass $m_{v}$. If this average is negative in condensing theory, the dynamics of a probing massive quark has no resemblance to its polarized massless counterpart. On the other hand, if the average is positive, the dynamics of the quark in question is under the influence of broken (valence or dynamical) chiral symmetry. The two kinds of behavior are distinguished simply by the sign of $S_{c h}$.

Elaborating on this, if the theory doesn't condense then $\rho_{c h}(\lambda) \leq 0$, and $S_{c h}\left(m_{v}\right) \leq 0$ for arbitrary $m_{v}$, consistently with chiral symmetry (valence or dynamical) not being broken. But in the condensing case $\rho_{c h}(\lambda)>0$ on $\left[0, \Lambda_{c h}\right)$, implying that $S_{c h}$ is positive for sufficiently small $m_{v}$. Indeed, the factor $m_{v} /\left(m_{v}^{2}+\lambda^{2}\right)$ plays role analogous to low-pass filter with passing spectral range controlled by $m_{v}$. In fact, there is $m_{c h}>0$ with $S_{c h}\left(m_{c h}\right)=0$, separating the "light quark" region driven mainly by broken chiral dynamics from the "heavy quark" region where chiral considerations play little role. The consequence of this for dynamical quark $m_{f} \in M$ is obvious: if $m_{f}<m_{c h}(M)$ then it is in the broken regime. Removing the reference to valence quarks, the above translates into the statement

$$
\text { flavor } m_{f} \in M \text { broken chiral } \quad \Longleftrightarrow \quad S_{c h}\left(m_{f}, M\right)>0
$$

Thus, the insight gained from the proposed association of SChSB with chiral polarization may provide a microscopic basis to judge the applicability of chiral perturbation theory. In particular, for $M=\left(m_{u}, m_{d}, m_{s}\right)$ of "real-world" QCD, one can inquire whether $S_{c h}\left(m_{s}, M\right)>0$.

It should be noted that densities $S$ and $S_{c h}$ involve a power cutoff divergence at non-zero quark mass [11], which needs to be subtracted at the spectral level for the above considerations to become meaningful. Discussion in this section thus implicitly assumed that the 


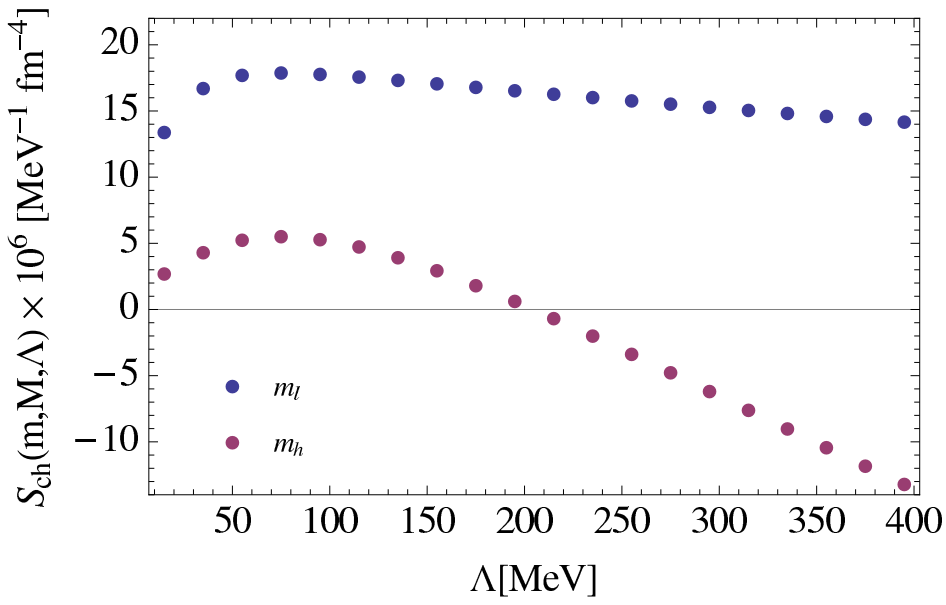

Figure 5: Cumulative chiral scalar density for "light" and "heavy" quark of $N_{f}=2+1$ QCD. See explanation in the text.

$\lambda$-dependent subtraction of mode density $\rho(\lambda) \rightarrow \rho_{\text {sub }}(\lambda)$, needed for proper spectral definition of $S$, is also used in the definition of $S_{c h}$. Related details will be discussed elsewhere [12].

In order to see the characteristic behavior, as well as to obtain the first estimate for the sign of $S_{c h}\left(m_{s}, M\right)$ in QCD, we present the result of a simplified calculation using the $m_{l} a=0.004$ dynamical ensemble of Sec. 4. Here the pion mass is still about twice the physical value, but the heavy quark $m_{h} a=0.03$ is at the strange mass. In Fig. 5 we show the plots of cumulative chiral scalar density $S_{c h}(m, M, \Lambda)$ defined by Eq. (44) via replacement $\int_{0}^{\infty} d \lambda \rightarrow \int_{0}^{\Lambda} d \lambda$. Note that both light $m \leftrightarrow m_{l}$ and heavy $m \leftrightarrow m_{h}$ cases are shown. Since overlap Dirac probe is used on domain-wall sea, the bare quark masses in the former need to be appropriately matched. In case of light quark we used the results of Ref. [13] for match based on the pion mass. The heavy mass was scaled up proportionally, which is an approximation. The $\lambda$-dependent subtraction in $\rho_{c h}$ was ignored but its effect is expected to be small at low-energy scales involved here.

As one can see, the behaviors for light and heavy quark are quite different. In the light case there is a very slow decrease of $S_{c h}(\Lambda)$ that will at larger scales become further moderated by the subtraction, and the positive $\Lambda \rightarrow \infty$ limit will presumably result. On the other hand, the density for the "strange quark" changes sign already at around $\Lambda=200$ $\mathrm{MeV}$, and the negativity appears rather robust even in light of the approximations involved.

8. The uses: Finite Temperature. The features of chiral symmetry discussed here may be of particular interest for studying QCD at finite temperature. Indeed, the characterization of changes in chiral properties of heated quarks and gluons is usually limited to monitoring of scalar densities $-\left\langle\bar{\psi} \psi_{f}\right\rangle_{M, T}=S\left(m_{f}, M, T\right)$ (or their susceptibility) as a function of temperature. However at non-zero quark masses, such as those of real world, chiral symmetry is explicitly broken and trends in $S\left(m_{f}, M, T\right)$ may not be most reflective of changes in the dynamics of the quark-gluon system. After all, crossover is currently a preferred scenario at physical quark masses [14], without a unique point separating definite qualitative behaviors.

However, if one takes a "dynamical mechanism" viewpoint, instead of the "thermodynamic" one, then there could be definite chirality-related properties that qualitatively change 
due to temperature. In the context proposed here, it is first of all natural to distinguish when the system supports density of chirally polarized quark modes. This feature is adjudicated by the positivity of $\Lambda_{c h}(M, T)$, and is equivalent to Dirac mode condensation as well as valence chiral symmetry breakdown. For arbitrary $M$ with $\Lambda_{c h}(M, T=0)>0$, we expect the existence of temperature $T_{c h}(M)>0$ such that

$$
\Lambda_{c h}(M, T)\left\{\begin{array}{lll}
>0 & \text { for } & T<T_{c h}(M) \\
=0 & \text { for } & T>T_{c h}(M)
\end{array}\right.
$$

Thus, $T_{c h}>0$ should exist in $\mathrm{N}_{f}=2+1$ QCD at arbitrary quark masses. We emphasize that $T_{c h}$ really characterizes the gluonic part in the dynamics of the massive quark-gluon system. Indeed, at $T>T_{c h}$ the relevant gauge backgrounds no longer support the feature (whatever it may be) allowing for chirally polarized modes to exist, and to facilitate broken dynamics of massless valence probe. When dynamical quarks are heavy, such change may not be crucial for their own dynamics, but this becomes so when they are light. Moreover, given the smooth approach to chiral limit seen in Fig. 3, $T_{c h}(M)$ probably marks the disappearance of the sought-after dynamical feature in glue, producing SChSB. Note also that, away from chiral limit, $\Lambda_{c h}(M, T)$ is a strictly non-local "order parameter" in terms of dynamical quark and gluon fields. Thus, its non-analytic nature at finite $T_{c h}(M)$ would not contradict the observed crossover behavior of standard thermal observables at physical point [14].

What about the characteristics relevant directly to dynamical quarks? In zero temperature QCD, chiral issues are mainly important because it is believed that "u" and "d" quarks are driven by broken chiral dynamics. It is then natural to expect that if given flavor(s) becomes no longer broken due to thermal agitation, the physical properties in the heated quark-gluon system qualitatively change. The degree of "broken quark dynamics" is controlled by $S_{c h}\left(m_{f}, M, T\right)$ in a manner specified by Eq. (7). For each flavor $f$ with $S_{c h}\left(m_{f}, M, T=0\right)>0$ we expect the existence of the temperature $T_{c h, f}(M)$ such that

$$
S_{c h}\left(m_{f}, M, T\right)\left\{\begin{array}{lll}
>0 & \text { for } & T<T_{c h, f}(M) \\
<0 & \text { for } & T>T_{c h, f}(M)
\end{array}\right.
$$

In summary, if the number of broken flavors at zero temperature is $0<N_{f, b} \leq N_{f}$, then there are $N_{f, b}+1$ transition temperatures characterizing chiral behavior, satisfying

$$
T_{c h} \geq T_{c h, 1} \geq T_{c h, 2} \geq \ldots \geq T_{c h, N_{f, b}}
$$

where flavors are assumed to be ordered by mass $m_{1} \leq m_{2} \leq \ldots \leq m_{N_{f}}$. The first relation becomes equality only when $m_{1}=0$ while the rest only when the corresponding pair of quarks is mass-degenerate. We emphasize again that these transitions may not correspond to phase transformations in the usual thermodynamic sense. Rather, they reflect qualitative changes in the dynamical behavior of participating quarks and gluons. In this way, the insight of chiral polarization offers a different perspective on QCD at finite temperature. 
Acknowledgments: We are indebted to RBC/UKQCD collaborations for sharing their dynamical configurations, and to Mike Lujan/ $\chi$ QCD collaboration for providing corresponding overlap eigenmodes. Thanks to Mike Creutz, Tamás Kovács and Stefan Sint for communications related to this project, and to Mingyang Sun for help with graphics. A. A. is supported in part by U.S. Department of Energy under the grant DE-FG02-95ER-40907 and by U.S. National Science Foundation under CAREER grant PHY-1151648. I.H. acknowledges the support by Department of Anesthesiology at the University of Kentucky.

\section{References}

[1] T. Banks and A. Casher, Nucl. Phys. B169, 125 (1980).

[2] I. Horváth, N. Isgur, J. McCune, H.B. Thacker, Phys. Rev. D65, 014502 (2002), hep-lat/0102003.

[3] A. Alexandru, T. Draper, I. Horváth, T. Streuer, Annals Phys. 326, 1941 (2011), arXiv:1009.4451.

[4] I. Horváth, hep-lat/0605008.

[5] H. Neuberger, Phys. Lett. B417 (1998) 141; Phys. Lett. B427 (1998) 353.

[6] R. Mawhinney, (RBC and UKQCD Collaborations) PoS LAT2009, 81 (2009), arXiv:0910.3194

[7] F. Karsch, Nucl. Phys. Proc. Suppl. 60A, 169 (1998), hep-lat/9706006.

[8] S. Chandrasekharan and N. Christ, Nucl. Phys. Proc. Suppl. 47, 527 (1996), hep-lat/9509095.

[9] T. Kovács, PoS LAT2008, 198 (2008), arXiv:0810.4763.

[10] A. Alexandru and I. Horváth, in preparation.

[11] P. Hernández, K. Jansen, L. Lellouch, Phys. Lett. B469 (1999) 198, hep-lat/9907022.

[12] A. Alexandru and I. Horváth, in progress.

[13] M. Lujan et al., Phys. Rev. D86, 014501 (2012), arXiv:1204.6256.

[14] Y. Aoki et al., Nature 443, 675 (2006), hep-lat/0611014. 